\title{
A comparison between two semantic deep learning frameworks for the autosomal dominant polycystic kidney disease segmentation based on magnetic resonance images
}

\author{
Vitoantonio Bevilacqua ${ }^{1 *}$, Antonio Brunetti ${ }^{1}$, Giacomo Donato Cascarano ${ }^{1}$, Andrea Guerriero1, \\ Francesco Pesce ${ }^{2}$, Marco Moschetta ${ }^{2}$ and Loreto Gesualdo ${ }^{2}$
}

From 2018 International Conference on Intelligent Computing (ICIC 2018) and Intelligent Computing and Biomedical Informatics (ICBI) 2018 conference

Wuhan and Shanghai, China. 15-18 August 2018, 3-4 November 2018

\begin{abstract}
Background: The automatic segmentation of kidneys in medical images is not a trivial task when the subjects undergoing the medical examination are affected by Autosomal Dominant Polycystic Kidney Disease (ADPKD). Several works dealing with the segmentation of Computed Tomography images from pathological subjects were proposed, showing high invasiveness of the examination or requiring interaction by the user for performing the segmentation of the images. In this work, we propose a fully-automated approach for the segmentation of Magnetic Resonance images, both reducing the invasiveness of the acquisition device and not requiring any interaction by the users for the segmentation of the images.
\end{abstract}

Methods: Two different approaches are proposed based on Deep Learning architectures using Convolutional Neural Networks (CNN) for the semantic segmentation of images, without needing to extract any hand-crafted features. In details, the first approach performs the automatic segmentation of images without any procedure for pre-processing the input. Conversely, the second approach performs a two-steps classification strategy: a first CNN automatically detects Regions Of Interest (ROls); a subsequent classifier performs the semantic segmentation on the ROls previously extracted.

Results: Results show that even though the detection of ROls shows an overall high number of false positives, the subsequent semantic segmentation on the extracted ROls allows achieving high performance in terms of mean Accuracy. However, the segmentation of the entire images input to the network remains the most accurate and reliable approach showing better performance than the previous approach.

Conclusion: The obtained results show that both the investigated approaches are reliable for the semantic segmentation of polycystic kidneys since both the strategies reach an Accuracy higher than 85\%. Also, both the investigated methodologies show performances comparable and consistent with other approaches found in literature working on images from different sources, reducing both the invasiveness of the analyses and the interaction needed by the users for performing the segmentation task.

Keywords: Deep learning, Convolutional neural network, Semantic segmentation, R-CNN, ADPKD, Magnetic resonance

\footnotetext{
*Correspondence: vitoantonio.bevilacqua@poliba.it

'Department of Electrical and Information Engineering (DEI), Polytechnic

University of Bari, Italy, Via Edoardo Orabona, 4, 70125 Bari, Italy

Full list of author information is available at the end of the article
}

(c) The Author(s). 2019 Open Access This article is distributed under the terms of the Creative Commons Attribution 4.0 International License (http://creativecommons.org/licenses/by/4.0/), which permits unrestricted use, distribution, and reproduction in any medium, provided you give appropriate credit to the original author(s) and the source, provide a link to the Creative Commons license, and indicate if changes were made. The Creative Commons Public Domain Dedication waiver (http://creativecommons.org/publicdomain/zero/1.0/) applies to the data made available in this article, unless otherwise stated. 


\section{Background}

Autosomal Dominant Polycystic Kidney Disease (ADPKD) is a hereditary disease characterised by the onset of renal cysts that lead to a progressive increase of the Total Kidney Volume (TKV) over time. Specifically, ADPKD is a genetic disorder in which the renal tubules become structurally abnormal, resulting in the development and growth of multiple cysts within the kidney parenchyma [1]. The mutation of two different genes characterises the disease. The ADPKD type I, which is caused by the PKD1 gene mutation, involves the $85-90 \%$ of the cases, usually affecting people older than 30 years. The mutation of the PKD2 gene, instead, leads to ADPKD type II (affecting the $10-15 \%$ of the cases), which mostly regards children developing cysts already when in the maternal uterus and die within a year. HConsidering the clinical characteristics of the patients with PKD1 or PKD2 mutations, they are the same, even though the latter mutation is associated with a milder clinical phenotype and a later onset of End-Stage Kidney Disease (ESKD). In all the cases, the size of cysts is extremely variable, ranging from some millimetres to $4-5$ centimetres [2].

Currently, there is not a specific cure for ADPKD and the TKV estimation over time allows to monitor the disease progression. Tolvaptan has been reported to slow the rate of cysts enlargement and, consequently, the progressive kidney function decline towards ESKD [3, 4]. Since all the actual pharmacological treatments aimed at slowing the growth of the cysts, the design of a non-invasive and accurate assessment of the renal volume is of fundamental importance for the estimation and assessment of the ADPKD progression over time.

There are several methods in the literature performing the TKV estimation; traditional methodologies, requiring imaging acquisitions, such as Computed Tomography (CT) and Magnetic Resonance (MR), include stereology and manual segmentation [5, 6]. Also, several studies tried to correlate this metric with body surface and area measurements in order to have a noninvasive estimation of TKV [7, 8]. Stereology consists in the superimposition of a square grid, with specific cell positions and spacing, on each slice of the volumetric acquisition (CT or MR). The bidimensional area obtained counting all the cells containing parts of the kidneys, interpolated with the other slices, considering the thickness of the acquisitions, allows obtaining the final three-dimensional volume. Manual segmentation, instead, requires the manual contouring of the kidney regions contained in every slice. Several tools supporting this task have been developed, introducing digital freehand contouring tools or interactive segmentation systems to assist the clinicians while delineating the region of interest.
Considering both the phenotyping of the disease and the introduced approaches, the segmentation of biomedical images of kidneys is a tricky and troublesome task, strictly dependent on the human operator performing the segmentation, also requiring expert training. In fact, comorbidities and the presence of cysts in neighbouring organs or contact surfaces make challenging achieving an accurate and standardised assessment of the TKV.

To reduce the limitations of the previous methodologies, both in time and performance, due to the manual interaction, several approaches for the semi-automatic segmentation of kidneys have been investigated such as the mid-slice or the ellipsoid methods, allowing to estimate the TKV starting from a reduced number of selected slices [9-11]. Although the reported methodologies are faster and more compliant than the previous ones, these are far from being accurate enough to be used in clinical protocols $[12,13]$.

In recent years, innovative approaches based on Deep Learning (DL) strategies have been introduced for the classification and segmentation of images. In details, deep architectures, such as Deep Neural Networks (DNNs) or Convolutional Neural Networks (CNNs), allowed to perform image classification tasks, detection of Regions Of Interest (ROIs) or semantic segmentation [14-17], reaching higher performance than traditional approaches [18]. The architecture of DL classifiers let avoiding the design of procedures for extracting hand-crafted features, as the classifier itself generally computes the most characteristic features automatically for each specific dataset. These peculiarities let DL approaches to be investigated in different fields, including medical imaging, signal processing or gene expression analysis [19-23].

Lastly, recent studies about imaging acquisitions for assessing kidneys growth suggested that MR should be preferred to other imaging techniques [24]. However, different research works allowed estimating TKV starting from CT images thanks to the higher availability of the acquisition devices and the more accurate and reliable measurement of TKV and the volume of cysts. On the other side, CT protocols for ADPKD are always contrast-enhanced using a contrast medium harmful for the health of the patient under examination; also, CT exposes the patients to ionising radiations. On these premises, the automatic, or semiautomatic, segmentation of images from MR acquisitions for improving the TKV estimation capabilities should be further investigated for improving the state-of-the-art performances.

Starting from a preliminary work performed on a small set of patients [25], we present two different approaches based on DL architectures to perform the automatic segmentation of kidneys affected by ADPKD 
starting from MR acquisitions. Specifically, we designed and evaluated several Convolutional Neural Networks, for discriminating the class of each pixel of the images, in order to perform their segmentation; Fig. 1 represents the corresponding workflow. Subsequently, we investigated the object detection approach using the Regions with CNN (R-CNN) technique [26] to automatically detect ROIs containing parts of the kidneys, with the aim to subsequently perform the semantic segmentation only on the extracted regions; Fig. 2 shows a representation of the workflow implemented in this approach.

\section{Methods}

\section{Patients and acquisition protocol}

From February to July 2017, 18 patients affected by ADPKD (mean age $31.25 \pm 15.52$ years) underwent Magnetic Resonance examinations for assessing the TKV. The acquisition protocol was carried out by the physicians from the Department of Emergency and Organ Transplantations (DETO) of the Bari University Hospital.

Examinations for the acquisition of the images were performed on a 1.5 Tesla MR device (Achieva, Philips Medical Systems, Best, The Netherlands) by using a four-channel breast coil. The protocol did not use contrast material intravenous injection and consisted of:

- Transverse and Coronal Short-TI Inversion Recovery (STIR) Turbo-Spin-Echo (TSE) sequences $(\mathrm{TR} / \mathrm{TE} / \mathrm{TI}=3.800 / 60 / 165 \mathrm{~ms}$, field of view $(\mathrm{FOV})=$ $250 \times 450 \mathrm{~mm}$ (AP $x \mathrm{RL})$, matrix $168 \times 300,50$ slices with $3 \mathrm{~mm}$ slice thickness and without gaps, 3 averages, turbo factor 23 , resulting in a voxel size of $1.5 \times 1.5 \times 3.0 \mathrm{~mm}^{3}$; sequence duration of $4.03 \mathrm{~min}$ );

- Transverse and Coronal T2-weighted TSE (TR/TE = $6.300 / 130 \mathrm{~ms}, \mathrm{FOV}=250 x 450 \mathrm{~mm}(\mathrm{AP} x \mathrm{RL})$, matrix $336 x 600,50$ slices with $3 \mathrm{~mm}$ slice thickness and without gaps, 3 averages, turbo factor 59, SENSE factor 1.7 , resulting in a voxel size of $0.75 \times 0.75 \times 3.0$ $\mathrm{mm}^{3}$; sequence duration of $3.09 \mathrm{~min}$ );

- Three-Dimensional (3D) T1-Weighted High Resolution Isotropic Volume Examination (THRIVE) sequence $(\mathrm{TR} / \mathrm{TE}=4.4 / 2.0 \mathrm{~ms}$, FOV $=250 x 450 x$ $150 \mathrm{~mm}$ (AP $x$ RL $x \mathrm{FH}$ ), matrix $168 x 300,100$ slices with $1.5 \mathrm{~mm}$ slice thickness, turbo factor 50, SENSE factor 1.6, data acquisition time of $1 \mathrm{~min} 30 \mathrm{~s}$ ).

In this work, only the coronal T2-Weighted TSE sequence only was considered for the processing and classification strategies. In order to have the segmentation ground truth for all the acquired images, our framework included a preliminary step allowing the radiologists to manually contour all the ROIs using a digital tool specifically designed and implemented for this task.

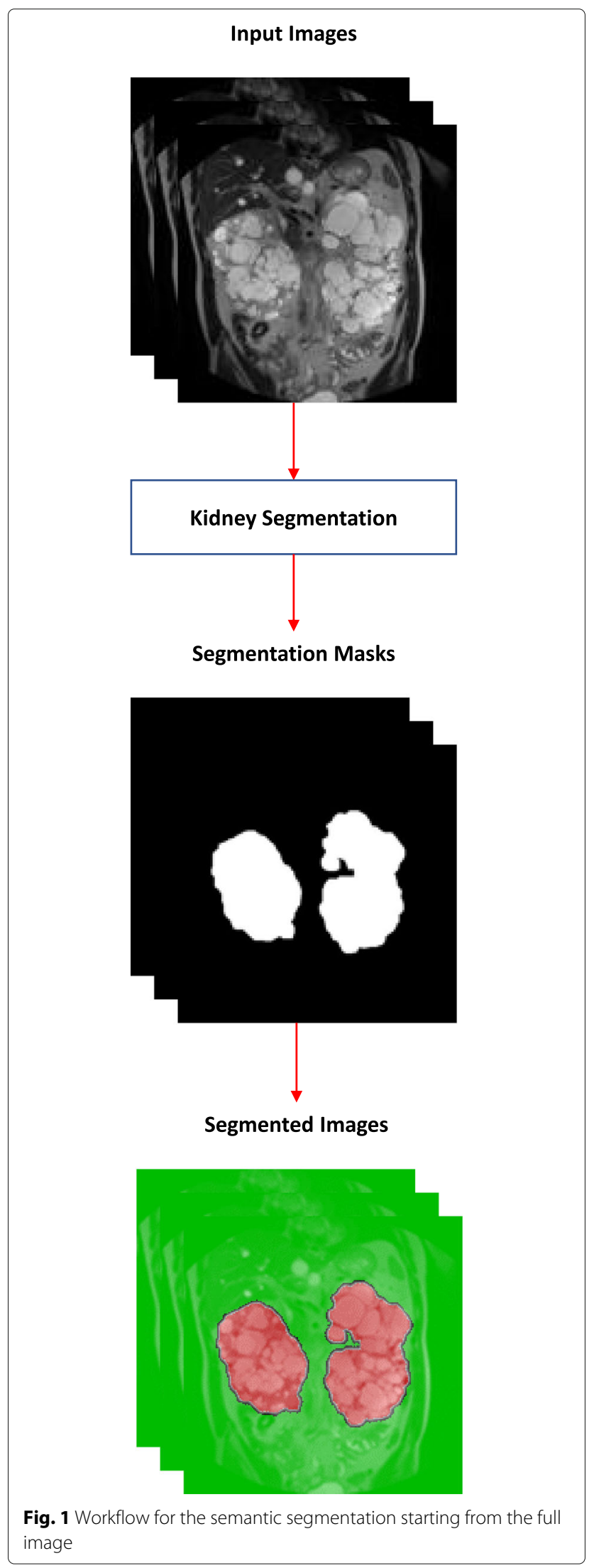




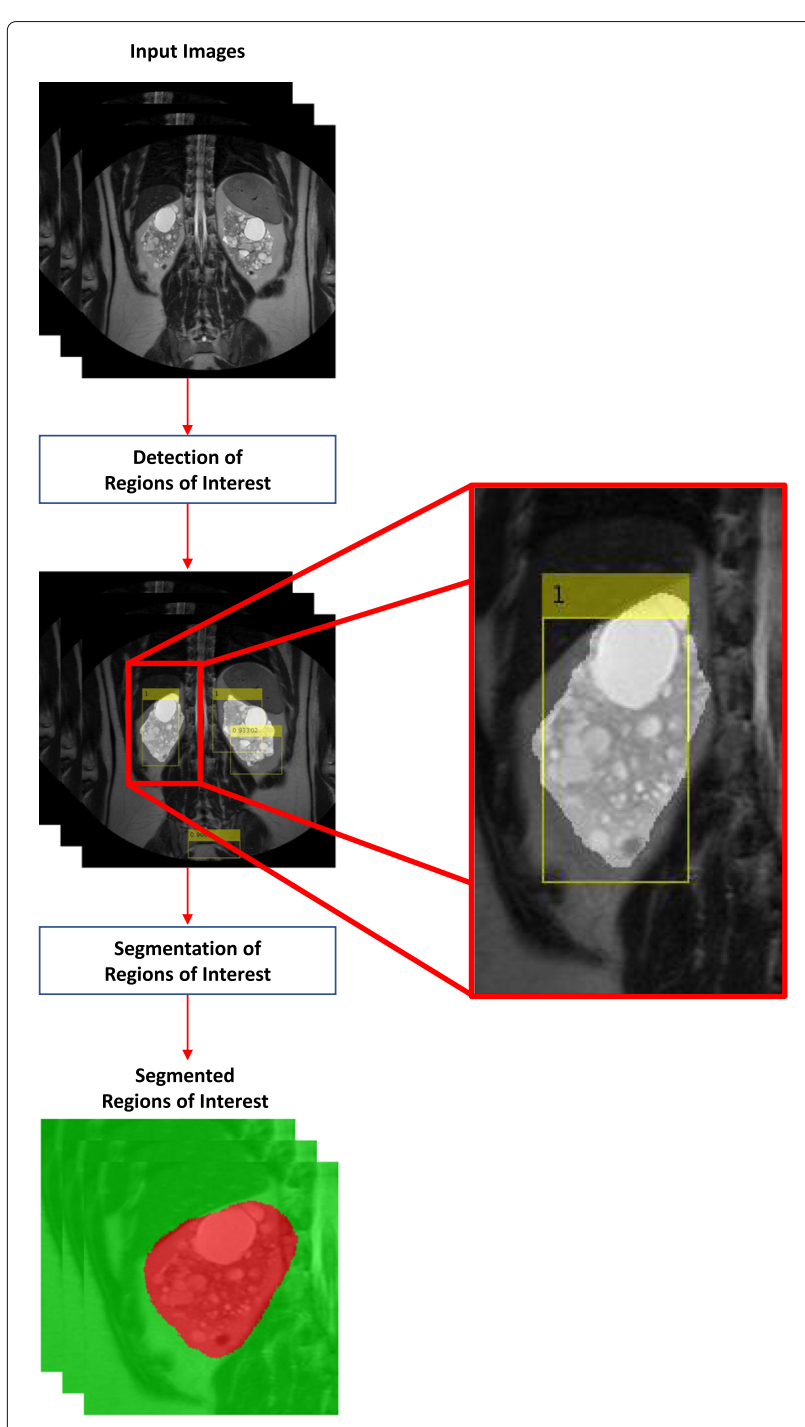

Fig. 2 Workflow for the semantic segmentation of ROls automatically detected with R-CNN

After the manual contouring of the kidneys, 526 images, with the corresponding labelled samples, constituted the working dataset; Fig. 3 represents an MR image with the corresponding labelled sample, where white pixels belong

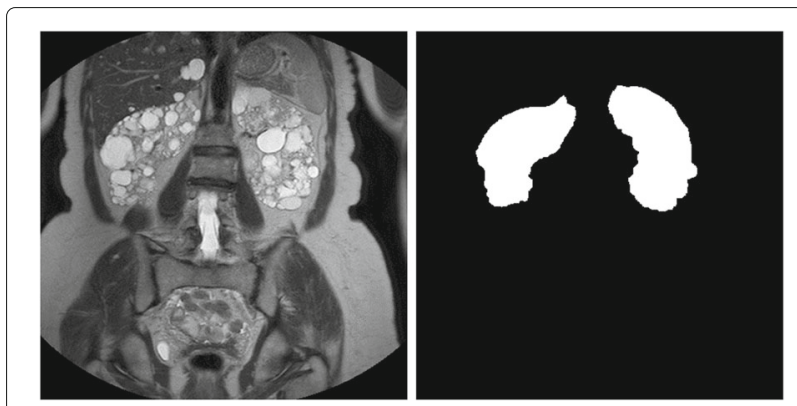

Fig. 3 Example of an input image segmented manually; left: the representation of a DICOM image in greyscale; right: the mask obtained after the manual contouring of the selected slice

to the kidneys whereas the black ones include the remaining parts of the image.

\section{Segmentation approaches}

Two different approaches based on DL techniques have been investigated to perform a fully-automated segmentation of polycystic kidneys without needing to design any procedure for the extraction of hand-crafted features. In details, the first approach allowed performing the semantic segmentation of the MR images, classifying each pixel belonging to the kidney or not; the second methodology, instead, allowed performing the detection of reduced areas containing the kidneys before their semantic segmentation.

\section{Semantic segmentation}

Semantic segmentation is a procedure allowing to perform the automatic classification of each pixel of images; thus, it is possible to classify each pixel of an image with a specific label. Although the segmentation of images is a well-established process in literature, counting a multitude of works and algorithms developed in several fields for different aims [27-29], the introduction and spread of DL architectures for performing this task, such as Convolutional Neural Networks, let image segmentation to regain interest in the scientific community [30,31].

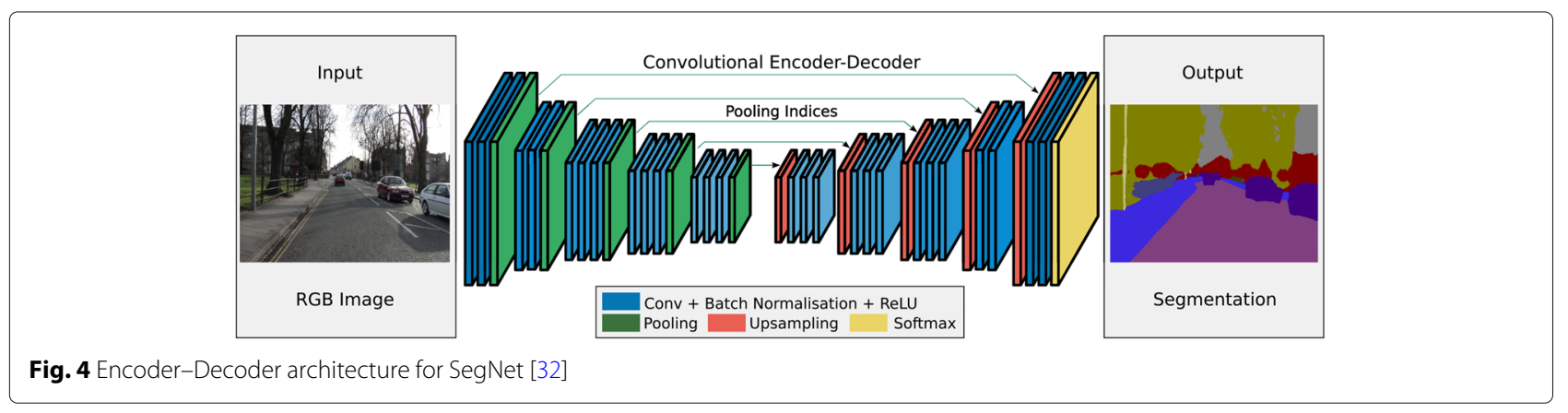


Table 1 Configurations designed and tested for the semantic segmentation of the full image

\begin{tabular}{|c|c|c|c|}
\hline Network ID & $\begin{array}{l}\text { Number of layers } \\
\text { per encoder }\end{array}$ & $\begin{array}{l}\text { Number of convolutional } \\
\text { filters per layer }\end{array}$ & Learner \\
\hline VGG-16 & {$\left[\begin{array}{lllllll}2 & 2 & 3 & 3 & 3\end{array}\right]$} & {$\left[\begin{array}{llllll}64 & 128 & 256 & 512 & 512]\end{array}\right.$} & ADAM \\
\hline$S-C N N-1$ & {$\left[\begin{array}{lllll}3 & 2 & 3 & 3 & 3\end{array}\right]$} & {$\left[\begin{array}{llllll}64 & 128 & 256 & 512 & 512\end{array}\right]$} & ADAM \\
\hline S-CNN-2 & {$\left[\begin{array}{lllll}3 & 2 & 3 & 3 & 3\end{array}\right]$} & {$\left[\begin{array}{llllll}96 & 128 & 256 & 512 & 512\end{array}\right]$} & ADAM \\
\hline
\end{tabular}

Each layer is a sequence of a convolutional layer, a batch normalization layer and a ReLu layer

According to different architectures designed in previous works, such as SegNet [32] and Fully Convolutional Network (FCN) [33], the CNNs performing semantic segmentation tasks show an encoder-decoder design, as the architecture represented in Fig. 4. Traditionally, this kind of classifier includes several encoders interspersed with pooling layers for downsampling the input; each encoder includes sequences of Convolutional layers, Normalisation layers and Linear layers. Based on the encoding part, there are specular decoders with up-sampling layers for reconstructing the input size. Finally, there are fully-connected neural units before the final classification layer able to label each pixel of the input image.

In this work, we designed and tested several CNNs architectures for the segmentation of the images. Since optimising the architecture of classifiers is still an open problem [34-36], often faced with evolutionary approaches, we decided to start from a well-known general CNN, the VGG-16 [37], and modify its structure varying several parameters.

These included the number of encoders (and decoders), the number of layers for each encoder, the number of convolutional filters for each layer and the learner
Table 2 Configurations designed and tested for the CNN in the $\mathrm{ROI}$ detector

\begin{tabular}{|c|c|c|c|}
\hline Network ID & $\begin{array}{l}\text { Number of layers } \\
\text { per encoder }\end{array}$ & $\begin{array}{l}\text { Number of } \\
\text { convolutional } \\
\text { filters per layer }\end{array}$ & Learner \\
\hline$R-C N N-1$ & [ $\left.\begin{array}{ll}3 & 3\end{array}\right]$ & [32 32] & SGDM \\
\hline R-CNN-2 & {$\left[\begin{array}{ll}1 & 1\end{array}\right]$} & [16 32] & SGDM \\
\hline$R-C N N-3$ & [3 3] & [64 32] & SGDM \\
\hline
\end{tabular}

Each layer is a sequence of a convolutional layer, and a ReLu layer

used during the training (i.e., SGDM - stochastic gradient descent with momentum, or ADAM [38]). All the investigated architectures included convolutional layers with kernels [3x3], stride [ [ 11 1] and padding [ [ $\left.\begin{array}{llll}1 & 1 & 1 & 1\end{array}\right]$ allowing to keep unchanged the dimensions of the input across each encoder; downsampling (and upsampling) was performed only in the max-pooling layers (upsampling layers for the decoder) having stride [l 2 2] and dimension [2x2].

The semantic segmentation of the input images took into account two classes: Kidney and Background. Considering the example reported in Fig. 3, the white pixels were labelled as Kidney, whereas the remaining pixels as Background.

For training the classifiers, also the dataset augmentation was performed according to recent works demonstrating the effectiveness of this procedure for improving the classification performance $[31,39,40]$; the following image transformations were randomly performed:

- horizontal shift in the range [-200; 200] pixels;

- horizontal flip;

- scaling with factor ranging in $[0.5 ; 4]$.
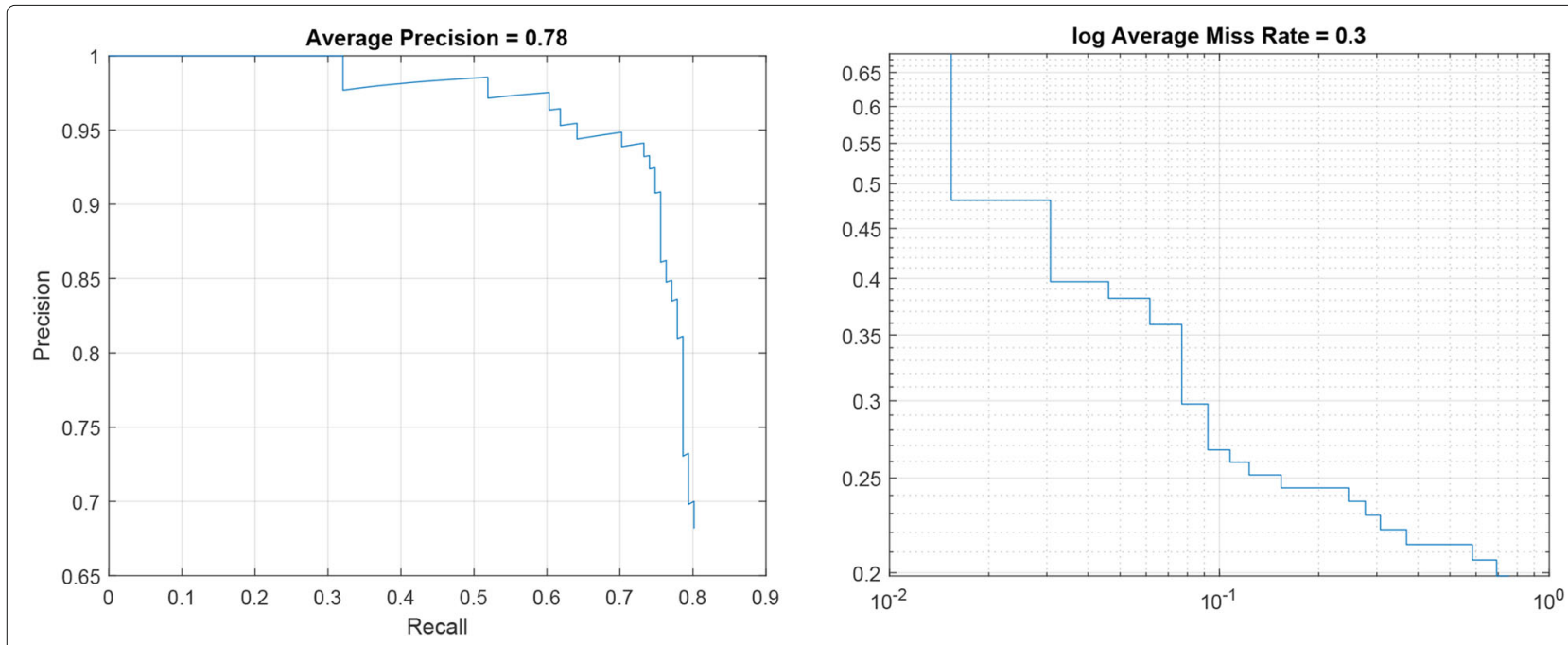

Fig. 5 Precision - Recall plot and log Average Miss rate for R-CNN-1 


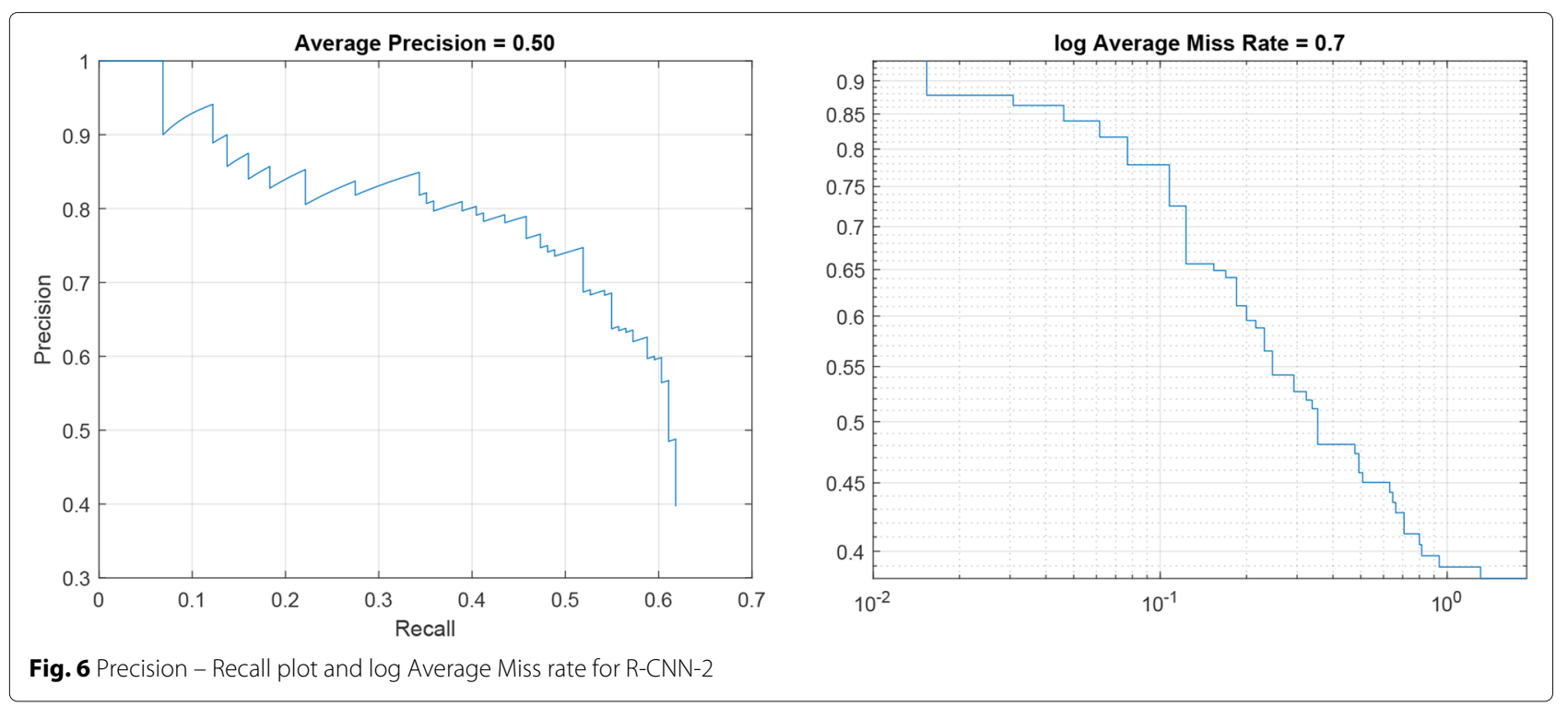

Table 1 reports the configurations designed and tested for performing this task in terms of number of layers per encoder, number of convolutional filters per layer and applied learner. The table reports only the three configurations showing the higher performance among all the investigated architectures.

\section{Regions with convolutional neural networks}

Due to the presence of cysts in the organs near the kidneys and very similar structures located near the area of interest, which may affect the segmentation performance, we investigated a second approach based on the object detection strategy using R-CNN. In this approach, we designed a classifier for performing the automatic detection of smaller regions inside each input image to subsequently segment according to the procedure described in the previous section.

Object detection is a technique for finding instances of specific classes in images or videos. Like the semantic segmentation, also the object detection is a well-established process in literature employed in different fields [41, 42]. According to the literature, the CNNs for object detection include a region proposal algorithm, often based on EdgeBoxes or Selective Search [43, 44], as a pre-processing step before running the classification algorithm. Traditional R-CNN and Fast R-CNN are the most employed techniques [26, 45]. Recently, Faster R-CNN was also introduced, addressing the region proposal mechanism using the $\mathrm{CNN}$ itself, thus making the region proposal a part of the $\mathrm{CNN}$ training and prediction steps [46].
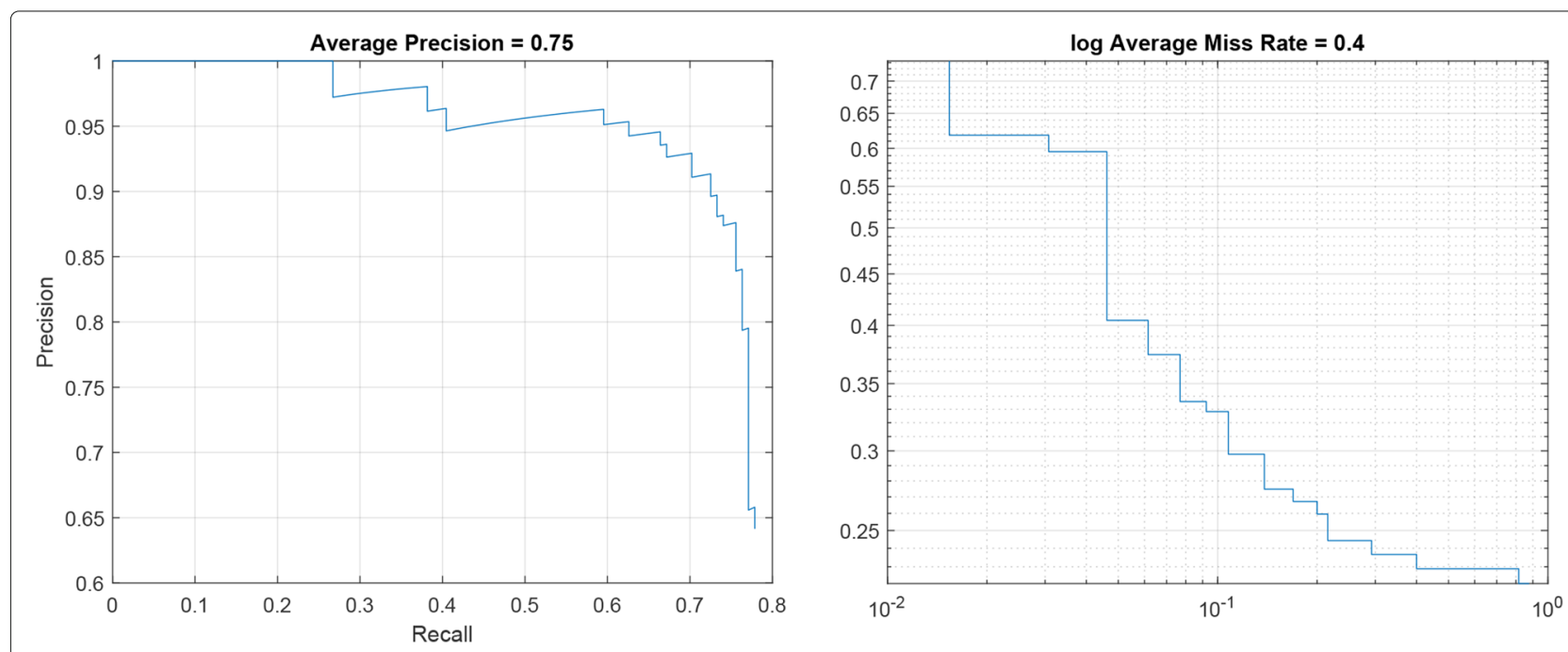

Fig. 7 Precision - Recall plot and log Average Miss rate for R-CNN-3 


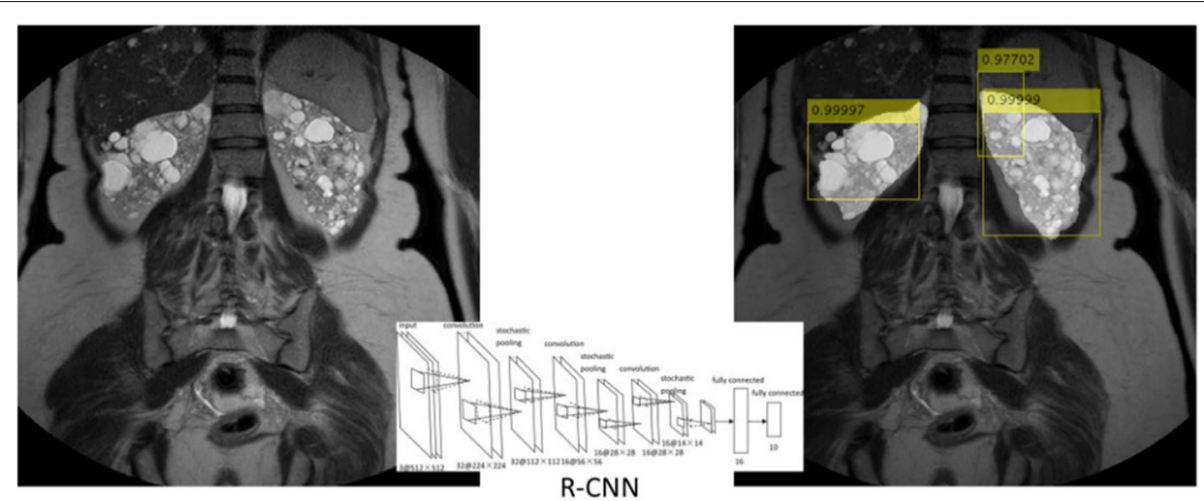

Fig. 8 Results from R-CNN classifier. Input image is on the left; the image on the right contains squares on the detected ROls, each one is associated with a score

FAs for the previous approach, we investigated several $\mathrm{CNN}$ architectures for detecting areas containing the kidney, considering the Fast R-CNN approach. For creating the ground truth, the manual contour of each kidney was enclosed in a rectangular bounding box and used for training the network. Differently for the $\mathrm{CNN}$ aimed at performing the semantic segmentation, these architectures have only the encoding part, where each encoder includes Convolutional layers and ReLu layers. Each encoder ends with a max-pooling layer to perform image sub-sampling (size [3x3] and stride [2 2]). At the end of the encoding part, there are two fully-connected layers before the final classification layer. Table 2 reports the configurations designed and tested for the detection purpose (in this case too, the table reports only the three configurations that reached the higher performance).

After designing the classifier for the automatic detection of the ROIs, the same architectures designed for the segmentation of the whole images (reported in Table 1), were considered for performing the semantic segmentation of the ROIs. Furthermore, since the detected ROIs might have different sizes, a rescaling procedure was performed to adapt all the ROIs to the size required by the $\mathrm{CNN}$ for the segmentation task. Images augmentation was performed, as well, considering the following image transformations:

- horizontal shift in the range $[-25 ; 25]$ pixels;

- vertical shift in the range $[-25 ; 25]$ pixels;

- horizontal flip;

- scaling with scale factor ranging in $[0.5 ; 1.1]$.

\section{Results}

This section reports the results for both the investigated approaches. In particular, we describe the performance obtained considering the R-CNN approach and subsequently, the results of the classifiers performing the semantic segmentation on both the full image and the ROIs automatically detected. The input dataset, which was constituted by MR images from 18 patients, was randomly split to create the training and test sets considering data from 15 and 3 patients, respectively. For improving the generalisation capabilities of the segmentation system, we performed a 5 -fold cross-validation for the training the classifiers. The final segmentation on the images from the test set was obtained through the majority voting computed among the segmentation results from each trained classifier.

We considered several metrics for evaluating the classifiers; all the reported results refer to the performance obtained evaluating the networks only on the test set. Accuracy (Eq. 1), Boundary F1 Score, or BF Score, (Eq. 2) and Jaccard Similarity Coefficient, or Intersection over Union - IoU, (Eq. 3)were computed considering the number of instances of True Positives $(T P)$, True Negatives $(T N)$, False Positives $(F P)$ and False Negatives $(F N)$, where the Positive label corresponds to a pixel belonging to the Kidney class for the semantic segmentation approach, or to a ROI correctly detected (confidence $>0.8$ ), for the $\mathrm{R}-\mathrm{CNN}$ approach.

Regarding the semantic segmentation, the BF Score measures how close the predicted boundary of an object matches the corresponding ground truth; it is defined as the harmonic mean of the Precision (Eq. 5) and Recall (Eq. 6) values. The resulting score spreads in the range $[0,1]$, from a bad to a good match. The Jaccard Similarity Coefficient, instead, is the ratio between the number of

Table 3 Performance indices for the classifiers working on MR images

\begin{tabular}{llll}
\hline Network ID & Mean accuracy & Weighted loU & Mean BF score \\
\hline VGG-16 & 0.88076 & 0.75288 & 0.41117 \\
S-CNN-1 & 0.88359 & 0.76294 & 0.38205 \\
S-CNN-2 & 0.79824 & 0.52781 & 0.38643 \\
\hline
\end{tabular}


Table 4 Normalized Confusion Matrix for VGG-16, S-CNN-1 and S-CNN-2 segmenting the MR images for the test set

\begin{tabular}{|c|c|c|c|c|c|c|c|}
\hline & & \multicolumn{2}{|l|}{ VGG-16 } & \multicolumn{2}{|l|}{ S-CNN-1 } & \multicolumn{2}{|c|}{ S-CNN-2 } \\
\hline & & \multicolumn{2}{|c|}{ True condition } & \multicolumn{2}{|c|}{ True condition } & \multicolumn{2}{|c|}{ True condition } \\
\hline & & Positive & Negative & Positive & Negative & Positive & Negative \\
\hline Predicted & Positive & 0.96629 & 0.20477 & 0.96146 & 0.19428 & 0.96595 & 0.21611 \\
\hline Condition & Negative & 0.03371 & 0.79523 & 0.03854 & 0.80572 & 0.03405 & 0.78389 \\
\hline
\end{tabular}

pixels belonging to the Positive class classified correctly $(T P)$ and the sum of the number of pixels belonging to the Positive class $(P=T P+F N)$ and the Negative pixels wrongly predicted as Positive $(F P)$. Regarding R-CNN performance, the Average Precision (Eq. 5) and the LogAverage Miss Rate were evaluated, considering the Miss Rate (MR) according to Eq. 4.

$$
\begin{aligned}
& \text { Accuracy }=\frac{T P+T N}{T P+T N+F P+F N} \\
& \text { Boundary F1 Score }=\frac{2 * \text { Precision } * \text { Recall }}{\text { Precision }+ \text { Recall }} \\
& \text { Jaccard Similarity Coefficient }=\frac{T P}{T P+F P+F N} \\
& \text { Miss Rate }=\frac{F N}{F N+T P}
\end{aligned}
$$

where:

$$
\begin{aligned}
& \text { Precision }=\frac{T P}{T P+F P} \\
& \text { Recall }=\frac{T P}{T P+F N}
\end{aligned}
$$

\section{R-CNN performance}

For each R-CNN architecture reported in Table 2, the Precision-Recall plot, showing the Precision obtained at different Recall values, and the Log-Average Miss Rate plot, reporting how varies the miss rate at different levels of FP per image are represented. Specifically, Figs. 5, 6 and 7 show the plots for R-CNN-1, R-CNN-2 and RCNN-3 respectively. Figure 8, instead, shows the result obtained performing the detection of kidneys on an image sample. As represented in the plots, the average Precision for R-CNN-1 and R-CNN-3 is higher than 0.75, also maintaining low the Log-Average Miss Rate.
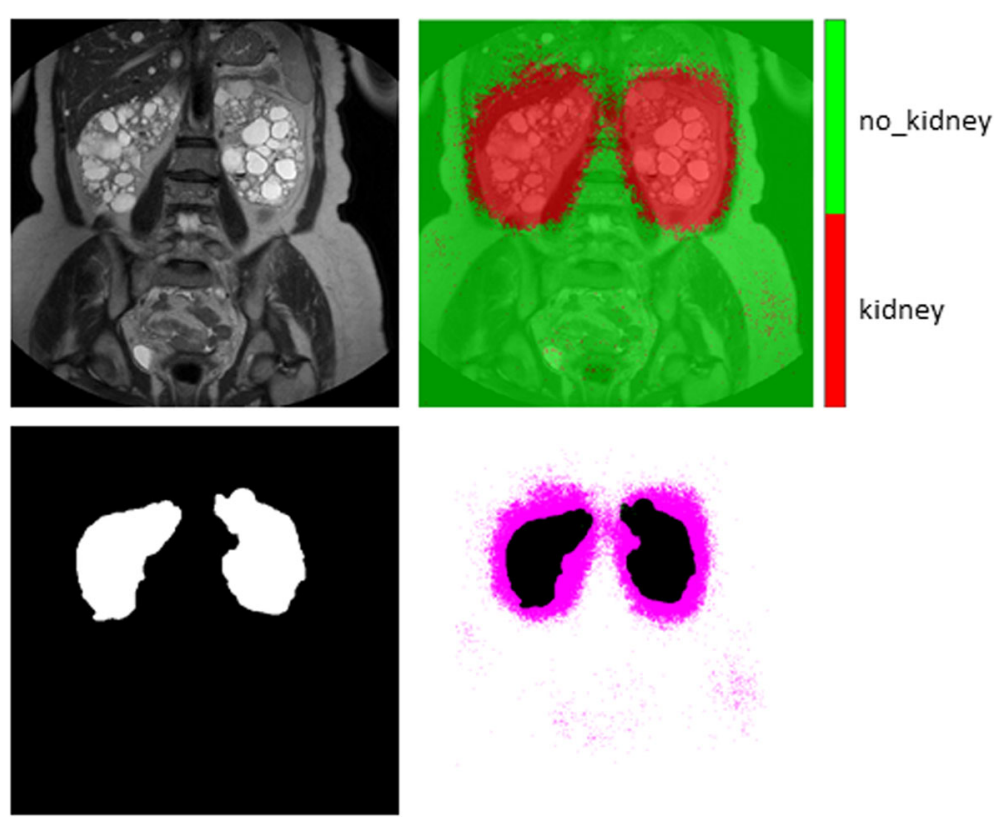

Fig. 9 Result of the semantic segmentation considering an image sample. Top left: the MR slice represented in greyscale; top right: the segmentation result; bottom left: the ground-truth mask; bottom right: superimposition of the segmentation result to the ground-truth mask 
Table 5 Performance indices for the classifiers working on the ROls

\begin{tabular}{llll}
\hline Network ID & Mean accuracy & Weighted loU & Mean BF score \\
\hline VGG-16 & 0.86016 & 0.75426 & 0.34828 \\
S-CNN-1 & 0.8726 & 0.8540 & 0.4332 \\
S-CNN-2 & 0.8550 & 0.82931 & 0.41515 \\
\hline
\end{tabular}

Since the aim of detecting ROIs was the identification of regions with fewer Background pixels, respect to the whole image, for the subsequent semantic segmentation step, $\mathrm{R}-\mathrm{CNN}-1$ revealed to be the best candidate among all the analysed architectures. In fact, it reached a Recall value of about 0.8 with the Precision higher than 0.65 , meaning that the classifier was able to detect the $80 \%$ of the ROIs containing the kidneys, but with a high number of False Positives. However, this was not a problem since the subsequent step of semantic segmentation would detect all the pixels belonging to the Kidney class.

\section{Semantic segmentation performance}

Concerning the semantic segmentation, this section reports the performance obtained for the segmentation of both MR images and ROIs. Specifically, Table 3 shows the results obtained for each of the $\mathrm{CNN}$ architectures performing the semantic segmentation of the MR image, without performing any image processing procedure. As reported in the table, the architecture achieving the highest performance for the semantic segmentation of the full image is the $S-C N N-1$, showing an Accuracy higher than $88 \%$.

The introduction of an additional layer into the first encoder of VGG-16 architecture allowed the network to create a set of features more significant and discriminative than those generated by the others, leading to more accurate classification performance. Conversely, increasing the number of convolutional filters in the first layer of the first encoder of $S-C N N-1$ did not improve the overall discrimination capabilities of the classifier. Table 4 reports the normalised confusion matrices obtained for the three considered cases in this approach, whereas Fig. 9 shows an example of the output generated by the implemented classifier performing the semantic segmentation of the MR images.

As for the segmentation of the whole MR images, Table 5 reports the performance indices for the semantic segmentation of the ROIs automatically detected by the $R-C N N-1$, which showed the optimal trade-off in detecting ROIs considering the miss rate. As for the previous case, the $S-C N N-1$ architecture allowed achieving the highest Accuracy in performing the semantic segmentation of the ROIs. Table 6 reports the normalised confusion matrices for all the classifiers investigated. Figure 10, instead, shows the results obtained for the semantic segmentation of ROI extracted from an image sample.

\section{Discussion}

In recent years, several works were proposed dealing with the segmentation of diagnostics images for assessing the ADPKD. Since the most used imaging procedure includes CT scans, most of the researches consider this kind of images in order to support the clinical assessment of the pathology. In some cases, the proposed approaches need minimum interaction by the user for the complete segmentation of the kidneys $[47,48]$. Also, some procedures in literature dealt with the fully-automated segmentation of the images, some of them based on DL strategies $[49,50]$.

However, the proposed approaches for the automatic segmentation show several limitations, including the invasiveness from the contrast medium used for enhancing CT acquisitions [51], or rather the necessity of having an a-priori knowledge for the correct processing of the images [52]. In order to reduce the invasiveness of the imaging analysis, a preliminary investigation proposing a fully automated approach for the segmentation of non-contrast-enhanced CT images was proposed very recently, showing good performance on a reduced cohort of patients [53].

In this work, instead, the developed classification systems allowed to reach performances of about $80 \%$ of Accuracy in performing the segmentation of MR images, without using any procedure for contrast enhancement. However, the segmentation of the entire MR image

Table 6 Normalized Confusion Matrix for VGG-16, S-CNN-1 and S-CNN-2 segmenting the ROls detected by the R-CNN-1 from the MR images of the test set

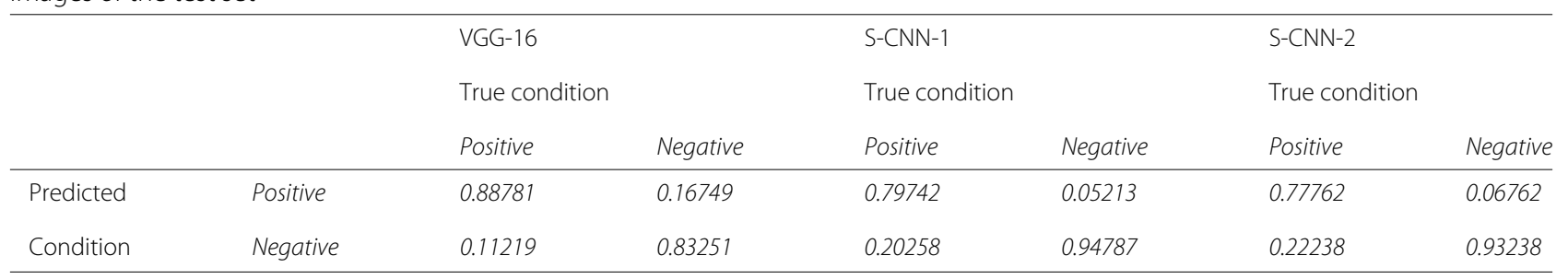




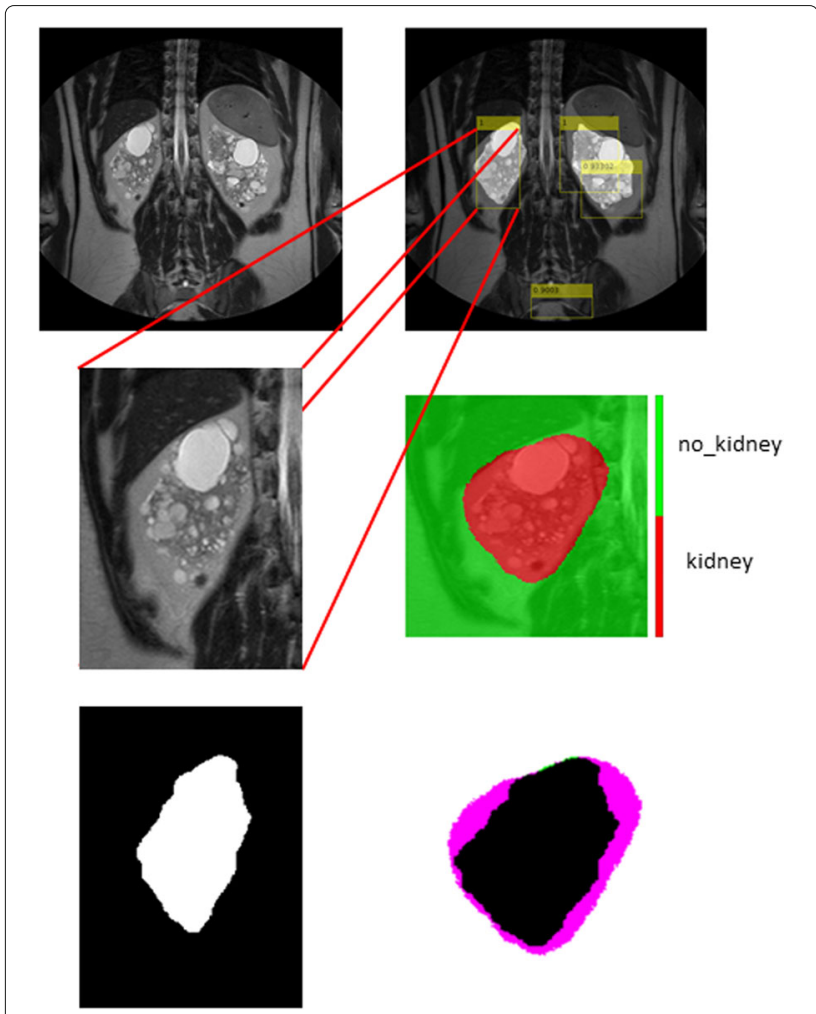

Fig. 10 Example result for $\mathrm{ROI}$ detection and semantic segmentation. Top left: the MR slice represented in greyscale; top right: the R-CNN detection result; middle left: one of the detected ROls; middle right the segmentation result; bottom left: the ground-truth mask for the considered ROl; bottom right: superimposition of the classification result to the ground-truth mask

revealed to be more reliable than those performed on the extracted ROIs. In fact, although the phase of extracting subregions from MR images showed an average Precision of $78 \%$, it could still not find areas of interest, thus missing regions belonging to the kidneys.

According to the analysed literature, the reported results are consistent with other precursory investigations dealing with MR images, including the preliminary results presented in [25] on a reduced cohort of patients. Also, the proposed approaches overcome the limitations shown by manual or semi-automatic procedures in segmenting kidneys affected by ADPKD for evaluating diagnostics and prognostics parameters. In addition, the proposed methodologies did not use any contrast medium, thus without any harmful or potentially lethal ionising radiation for the patients.

\section{Conclusions}

In this work, we investigated two strategies performing the automatic segmentation of MR images from people affected by ADPKD based on DL architectures. Both the designed strategies considered several Convolutional
Neural Networks for classifying, between Kidney or Backgroud, all the pixels in the images.

In the first approach, we trained, validated and tested the classifiers considering the full MR image as input, without performing any procedure of image preprocessing. The second methodology, instead, investigated the object detection approach using the Regions with CNN (R-CNN) technique for firstly detecting ROIs containing parts of the kidneys. Subsequently, we employed (trained, validated and tested) the CNNs considered in the previous approach to perform the semantic segmentation on the ROIs automatically extracted by the R-CNN showing the most reliable performance.

The obtained results show that both the approaches are comparable and consistent with other methodologies reported in the literature, but dealing with images from different sources, such as CT scans. Also, the proposed approaches may be considered reliable methods to perform a fully-automated segmentation of kidneys affected by ADPKD.

In the future, the interaction among Deep Learning strategies and image processing techniques will be further investigated to improve the performance reached by the actual classifiers. Moreover, evolutionary approaches for optimising the topology of classifiers, or their hyperparameters, will also be explored considering the acquired images in a three-dimensional way.

\section{Abbreviations}

ADPKD: Autosomal dominant polycystic kidney disease; CNN: Convolutional neural network; CT: Computed tomography; DL: Deep learning; DNN: Deep neural network; ESKD: End-stage kidney disease; MR: Magnetic resonance; R-CNN: Regions with convolutional neural network; ROl: Region of interest; TKV: Total kidney volume

\section{Acknowledgements}

The authors wish to thanks the colleagues from University of Bari "Aldo Moro" who provided insight and expertise that greatly assisted the research.

\section{About this supplement}

This article has been published as part of BMC Medical Informatics and Decision Making Volume 19 Supplement 9, 2019: Proceedings of the 2018 International Conference on Intelligent Computing (ICIC 2018) and Intelligent Computing and Biomedical Informatics (ICBI) 2018 conference: medical informatics and decision making. The full contents of the supplement are available online at https:// bmcmedinformdecismak.biomedcentral.com/articles/supplements/volume19-supplement-9.

\section{Authors' contributions}

$V B, L G$ and $M M$ conceived the study and participated in its design and coordination. $\mathrm{AB}$ and GDC designed the classifiers and carried out the data classification and segmentation. MM and VB organized the enrolment of the patients and the acquisition of the data and then validated the final results. VB and $A B$ drafted the manuscript and then all the authors read and approved the final manuscript.

\section{Funding}

Publication costs have been partially funded by the PON MISE 2014-2020 "HORIZON 2020" program, project PRE.MED.: Innovative and integrated platform for the predictive diagnosis of the risk of progression of chronic kidney disease, targeted therapy and proactive assistance for patients with autosomal dominant polycystic genetic disease. 


\section{Availability of data and materials}

The dataset employed for the current study is not publicly available due to restrictions associated with the anonymity of participants but could be made available from the corresponding author on reasonable request.

\section{Ethics approval and consent to participate}

The experimental procedures were conducted in accordance with the Declaration of Helsinki. All participants provided written informed consent.

\section{Consent for publication}

Not applicable.

\section{Competing interests}

The authors declare that they have no competing interests.

\section{Author details}

${ }^{1}$ Department of Electrical and Information Engineering (DEI), Polytechnic University of Bari, Italy, Via Edoardo Orabona, 4, 70125 Bari, Italy. ${ }^{2}$ D.E.T.O. University of Bari Medical School, Piazza Giulio Cesare, 11, 70124 Bari, Italy.

\section{Published: 12 December 2019}

\section{References}

1. Grantham JJ. Autosomal dominant polycystic kidney disease. N Engl J Med. 2008;359(14):1477-85. https://doi.org/10.1056/NEJMcp0804458.

2. Harris PC, Bae KT, Rossetti S, Torres VE, Grantham JJ, Chapman AB, Guay-Woodford LM, King BF, Wetzel LH, Baumgarten DA, Kenney PJ, Consugar M, KlahrS, Bennett WM, Meyers CM, Zhang Q, Thompson PA, Zhu F, Miller JP. Cyst number but not the rate of cystic growth is associated with the mutated gene in autosomal dominant polycystic kidney disease. J Am Soc Nephrol. 2006;17(11):3013-9. https://doi.org/10.1681/ASN.2006080835.

3. Torres VE, Chapman AB, Devuyst O, Gansevoort RT, Grantham JJ, Higashihara E, Perrone RD, Krasa HB, Ouyang J, Czerwiec FS. Tolvaptan in patients with autosomal dominant polycystic kidney disease. N Engl J Med. 2012;367(25):2407-18. https://doi.org/10.1056/NEJMoa1205511.

4. Irazabal MV, Torres VE, Hogan MC, Glockner J, King BF, Ofstie TG, Krasa HB, Ouyang J, Czerwiec FS. Short-term effects of tolvaptan on renal function and volume in patients with autosomal dominant polycystic kidney disease. Kidney Int. 2011;80(3):295-301. https://doi.org/10.1038/ki.2011.119.

5. Bae KT, Commean PK, Lee J. Volumetric measurement of renal cysts and parenchyma using mri: Phantoms and patients with polycystic kidney disease. J Comput Assist Tomogr. 2000;24(4):614-9. https://doi.org/10. 1097/00004728-200007000-00019.

6. King BF, Reed JE, Bergstralh EJ, Sheedy PF, Torres VE. Quantification and longitudinal trends of kidney, renal cyst, and renal parenchyma volumes in autosomal dominant polycystic kidney disease. J Am Soc Nephrol. 2000;11(8):1505-11.

7. Vauthey JN, Abdalla EK, Doherty DA, Gertsch P, Fenstermacher MJ, Loyer EM, Lerut J, Materne R, Wang X, Encarnacion A, Herron D, Mathey C, Ferrari G, Charnsangavej C, Do KA, Denys A. Body surface area and body weight predict total liver volume in western adults. Liver Transplant. 2002;8(3):233-40. https://doi.org/10.1053/jlts.2002.31654.

8. Emamian SA, Nielsen MB, Pedersen JF, Ytte L. Kidney dimensions at sonography: Correlation with age, sex, and habitus in 665 adult volunteers. Am J Roentgenol. 1993;160(1):83-6. https://doi.org/10.2214/ ajr.160.1.8416654.

9. Higashihara E, Nutahara K, Okegawa T, Tanbo M, Hara H, Miyazaki I, Kobayasi K, Nitatori T. Kidney volume estimations with ellipsoid equations by magnetic resonance imaging in autosomal dominant polycystic kidney disease. Nephron. 2015;129(4):253-62. https://doi.org/ 10.1159/000381476.

10. Irazabal MV, Rangel $L J$, Bergstralh EJ, Osborn SL, Harmon AJ, Sundsbak $J$, Bae KT, Chapman AB, Grantham JJ, Mrug M, Hogan MC, El-Zoghby ZM, Harris PC, Erickson BJ, King BF, Torres VE. Imaging classification of autosomal dominant polycystic kidney disease: A simple model for selecting patients for clinical trials. J Am Soc Nephrol. 2015;26(1):160-72. https://doi.org/10.1681/ASN.2013101138.

11. Bae KT, Tao C, Wang J, Kaya D, Wu Z, Bae JT, Chapman AB, Torres VE, Grantham JJ, Mrug M, Bennett WM, Flessner MF, Landsittel DP. Novel approach to estimate kidney and cyst volumes using mid-slice magnetic resonance images in polycystic kidney disease. Am J Nephrol. 2013;38(4): 333-41. https://doi.org/10.1159/000355375. NIHMS150003.

12. Grantham JJ, Torres VE. The importance of total kidney volume in evaluating progression of polycystic kidney disease. Nat Rev Nephrol. 2016;12(11):667-77. https://doi.org/10.1038/nrneph.2016.135. 15334406.

13. Grantham JJ, Torres VE, Chapman AB, Guay-Woodford LM, Bae KT, King BF, Wetzel LH, Baumgarten DA, Kenney PJ, Harris PC, Klahr S, Bennett WM, Hirschman GN, Meyers CM, Zhang X, Zhu F, Miller JP. Volume progression in polycystic kidney disease. N Engl J Med. 2006;354(20): 2122-30. https://doi.org/10.1056/NEJMoa054341.

14. Brunetti A, Carnimeo L, Trotta GF, Bevilacqua V. Computer-assisted frameworks for classification of liver, breast and blood neoplasias via neural networks: A survey based on medical images. Neurocomputing. 2019;335:274-98. https://doi.org/10.1016/j.neucom.2018.06.080.

15. Biswas M, Kuppili V, Saba L, Edla D, Suri H, Cuadrado-Godia E, Laird J, Marinhoe R, Sanches J, Nicolaides A, et al. State-of-the-art review on deep learning in medical imaging. Front Biosci (Landmark Ed). 2019;24:392-426.

16. Akkus Z, Galimzianova A, Hoogi A, Rubin DL, Erickson BJ. Deep learning for brain MRI segmentation: State of the art and future directions. J Digit Imaging. 2017;30(4):449-59. https://doi.org/10.1007/s10278-017-9983-4.

17. Lecun Y, Bengio Y, Hinton G. Deep learning. Nature. 2015;521(7553): 436-44. https://doi.org/10.1038/nature14539.

18. Bevilacqua V, Brunetti A, Guerriero A, Trotta GF, Telegrafo M, Moschetta M. A performance comparison between shallow and deeper neural networks supervised classification of tomosynthesis breast lesions images. Cogn Syst Res. 2019;53:3-19. https://doi.org/10.1016/j.cogsys. 2018.04.011.

19. Shen Z, Bao W, Huang D-S. Recurrent neural network for predicting transcription factor binding sites. Sci Rep. 2018;8(1):15270.

20. Deng S-P, Cao S, Huang D-S, Wang Y-P. Identifying stages of kidney renal cell carcinoma by combining gene expression and dna methylation data. IEEE/ACM Trans Comput Biol Bioinforma. 2017;14(5):1147-53.

21. Yi H-C, You Z-H, Huang D-S, Li X, Jiang T-H, Li L-P. A deep learning framework for robust and accurate prediction of ncrna-protein interactions using evolutionary information. Mol Therapy-Nucleic Acids 2018;11:337-44.

22. Litjens $G$, Kooi T, Bejnordi BE, Setio AAA, Ciompi F, Ghafoorian M, van der Laak JAWM, van Ginneken B, Sánchez Cl. A survey on deep learning in medical image analysis. Med Image Anal. 2017;42:60-88. https://doi.org/10.1016/j.media.2017.07.005. 1702.05747.

23. Schmidhuber J. Deep learning in neural networks: An overview. Neural Netw. 2015;61:85-117. https://doi.org/10.1016/j.neunet.2014.09.003. 1404.7828 .

24. Magistroni R, Corsi C, Martí T, Torra R. A review of the imaging techniques for measuring kidney and cyst volume in establishing autosomal dominant polycystic kidney disease progression. Am J Nephrol. 2018;48(1):67-78.

25. Bevilacqua V, Brunetti A, Cascarano GD, Palmieri F, Guerriero A, Moschetta M. A deep learning approach for the automatic detection and segmentation in autosomal dominant polycystic kidney disease based on magnetic resonance images. In: Huang D, Jo K, Zhang X, editors. Intelligent Computing Theories and Application - 14th International Conference, ICIC 2018, Wuhan, China, August 15-18, 2018, Proceedings, Part II. Lecture Notes in Computer Science, vol. 10955. Cham: Springer; 2018. p. 643-9. https://doi.org/10.1007/978-3-319-95933-7_73.

26. Girshick R, Donahue J, Darrell T, Malik J. Rich feature hierarchies for accurate object detection and semantic segmentation. In: Proceedings of the 2014 IEEE Conference on Computer Vision and Pattern Recognition. CVPR '14. Washington: IEEE Computer Society; 2014. p. 580-7. https://doi. org/10.1109/CVPR.2014.81.

27. Bevilacqua V, Dimauro G, Marino F, Brunetti A, Cassano F, Maio AD, Nasca E, Trotta GF, Girardi F, Ostuni A, Guarini A. A novel approach to evaluate blood parameters using computer vision techniques. In: 2016 IEEE International Symposium on Medical Measurements and Applications, MeMeA 2016, Benevento, Italy, May 15-18, 2016; 2016. p. 1-6. https://doi.org/10.1109/MeMeA.2016.7533760.

28. Bevilacqua V, Pietroleonardo N, Triggiani V, Brunetti A, Di Palma AM, Rossini M, Gesualdo L. An innovative neural network framework to classify blood vessels and tubules based on haralick features evaluated in histological images of kidney biopsy. Neurocomputing. 2017;228:143-53. https://doi.org/10.1016/j.neucom.2016.09.091. 
29. Bevilacqua V, Brunetti A, Trotta GF, Dimauro G, Elez K, Alberotanza V, Scardapane A. A novel approach for hepatocellular carcinoma detection and classification based on triphasic CT protocol. In: 2017 IEEE Congress on Evolutionary Computation, CEC 2017, Donostia, San Sebastián, Spain, June 5-8, 2017; 2017. p. 1856-63. https://doi.org/10.1109/CEC.2017. 7969527.

30. Garcia-Garcia A, Orts-Escolano S, Oprea S, Villena-Martinez V, Rodríguez JG. A review on deep learning techniques applied to semantic segmentation. CoRR. 2017;abs/1704.06857:. 1704.06857.

31. Bevilacqua $V$, Altini $D$, Bruni $M$, Riezzo M, Brunetti $A$, Loconsole $C$, Guerriero A, Trotta GF, Fasano R, Pirchio MD, Tartaglia C, Ventrella E, Telegrafo M, Moschetta M. A supervised breast lesion images classification from tomosynthesis technique. In: Huang D, Jo K, FigueroaGarcía JC, editors. Intelligent Computing Theories and Application - 13th International Conference, ICIC 2017, Liverpool, UK, August 7-10, 2017, Proceedings, Part II. Lecture Notes in Computer Science, vol. 10362. Cham: Springer; 2017. p. 483-9. https://doi.org/10.1007/978-3-319-63312-1_42.

32. Badrinarayanan V, Kendall A, Cipolla R. Segnet: A deep convolutional encoder-decoder architecture for image segmentation. CoRR. 2015;abs/1511.00561:. 1511.00561.

33. Brostow GJ, Fauqueur J, Cipolla R. Semantic object classes in video: A high-definition ground truth database. Pattern Recogn Lett. 2009;30(2): 88-97. https://doi.org/10.1016/j.patrec.2008.04.005.

34. Buongiorno D, Barsotti M, Barone F, Bevilacqua V, Frisoli A. A linear approach to optimize an emg-driven neuromusculoskeletal model for movement intention detection in myo-control: A case study on shoulder and elbow joints. Front Neurorobotics. 2018;12:74.

35. Bortone I, Trotta GF, Brunetti A, Cascarano GD, Loconsole C, Agnello N, Argentiero A, Nicolardi G, Frisoli A, Bevilacqua V. A novel approach in combination of $3 \mathrm{~d}$ gait analysis data for aiding clinical decision-making in patients with parkinson's disease. In: Intelligent Computing Theories and Application. ICIC 2017. Lecture Notes in Computer Science, vol 10362. Cham: Springer; 2017. p. 504-14. https://doi.org/10.1007/978-3-31963312-1_44.

36. Bevilacqua V, Uva AE, Fiorentino M, Trotta GF, Dimatteo M, Nasca E, Nocera AN, Cascarano GD, Brunetti A, Caporusso N, et al. A comprehensive method for assessing the blepharospasm cases severity. In: Recent Trends in Image Processing and Pattern Recognition. RTIP2R 2016. Communications in Computer and Information Science, vol 709. Singapore: Springer; 2016. p. 369-81. https://doi.org/10.1007/978-98110-4859-3_33.

37. Simonyan K, Zisserman A. Very deep convolutional networks for large-scale image recognition. arXiv preprint arXiv:1409.1556. 2014.

38. Kingma DP, Ba J. Adam: A method for stochastic optimization. arXiv preprint arXiv:1412.6980. 2014

39. Wong SC, Gatt A, Stamatescu V, McDonnell MD. Understanding data augmentation for classification: When to warp? In: 2016 International Conference on Digital Image Computing: Techniques and Applications, DICTA 2016, Gold Coast, Australia, November 30 - December 2, 2016; 2016. p. 1-6. https://doi.org/10.1109/DICTA.2016.7797091.

40. Xu Y, Jia R, Mou L, Li G, Chen Y, Lu Y, Jin Z. Improved relation classification by deep recurrent neural networks with data augmentation. In: COLING 2016, 26th International Conference on Computational Linguistics, Proceedings of the Conference: Technical Papers, December 11-16. Japan: ACL; 2016. p. 1461-70.

41. Brunetti A, Buongiorno D, Trotta GF, Bevilacqua V. Computer vision and deep learning techniques for pedestrian detection and tracking: A survey. Neurocomputing. 2018;300:17-33. https://doi.org/10.1016/j.neucom. 2018.01.092.

42. Kulchandani JS, Dangarwala KJ. Moving object detection: Review of recent research trends. In: 2015 International Conference on Pervasive Computing (ICPC), Pune. IEEE; 2015. p. 1-5. https://doi.org/10.1109/ PERVASIVE.2015.7087138.

43. Zitnick CL, Dollár P. Computer Vision - ECCV 2014 - 13th European Conference, Zurich, Switzerland, September 6-12, 2014, Proceedings, Part V. Lecture Notes in Computer Science, vol. 8693. In: Fleet DJ, Pajdla T, Schiele B, Tuytelaars T, editors. Cham: Springer; 2014. p. 391-405. https:// doi.org/10.1007/978-3-319-10602-1_26.
44. Uijlings JRR, van de Sande KEA, Gevers T, Smeulders AWM. Selective search for object recognition. Int J Comput Vis. 2013;104(2):154-71. https://doi.org/10.1007/s11263-013-0620-5.

45. Girshick RB. Fast R-CNN. In: 2015 IEEE International Conference on Computer Vision, ICCV 2015, Santiago, Chile, December 7-13, 2015; 2015. p. 1440-8. https://doi.org/10.1109/ICCV.2015.169.

46. Ren S, He K, Girshick RB, Sun J. Faster R-CNN: towards real-time object detection with region proposal networks. IEEE Trans Pattern Anal Mach Intell. 2017;39(6):1137-49. https://doi.org/10.1109/TPAMI.2016.2577031.

47. Sharma K, Peter L, Rupprecht C, Caroli A, Wang L, Remuzzi A, Baust M, Navab N. Semi-automatic segmentation of autosomal dominant polycystic kidneys using random forests. arXiv e-prints. 20151510-06915. 1510.06915.

48. Kline TL, Edwards ME, Korfiatis P, Akkus Z, Torres VE, Erickson BJ. Semiautomated segmentation of polycystic kidneys in t2-weighted $\mathrm{mr}$ images. Am J Roentgenol. 2016;207(3):605-13.

49. Kline TL, Korfiatis P, Edwards ME, Blais JD, Czerwiec FS, Harris PC, King BF, Torres VE, Erickson BJ. Performance of an artificial multi-observer deep neural network for fully automated segmentation of polycystic kidneys. J Digit Imaging. 2017;30(4):442-8.

50. Kline $T L$, Korfiatis P, Edwards ME, Warner JD, Irazabal MV, King BF, Torres VE, Erickson BJ. Automatic total kidney volume measurement on follow-up magnetic resonance images to facilitate monitoring of autosomal dominant polycystic kidney disease progression. Nephrol Dial Transplant. 2015;31(2):241-8.

51. Sharma K, Rupprecht C, Caroli A, Aparicio MC, Remuzzi A, Baust M, Navab N. Automatic segmentation of kidneys using deep learning for total kidney volume quantification in autosomal dominant polycystic kidney disease. Sci Rep. 2017;7(1):2049.

52. Kim Y, Ge Y, Tao C, Zhu J, Chapman AB, Torres VE, Yu ASL, Mrug M, Bennett WM, Flessner MF, Landsittel DP, Bae KT, for the Consortium for Radiologic Imaging Studies of Polycystic Kidney Disease (CRISP). Automated segmentation of kidneys from mr images in patients with autosomal dominant polycystic kidney disease. Clin J Am Soc Nephrol. 2016;11(4):576-84. https://doi.org/10.2215/CJN.08300815.

53. Turco D, Valinoti M, Martin EM, Tagliaferri C, Scolari F, Corsi C. Fully automated segmentation of polycystic kidneys from noncontrast computed tomography: A feasibility study and preliminary results. Acad Radiol. 2018;25(7):850-5.
Ready to submit your research? Choose BMC and benefit from:

- fast, convenient online submission

- thorough peer review by experienced researchers in your field

- rapid publication on acceptance

- support for research data, including large and complex data types

- gold Open Access which fosters wider collaboration and increased citations

- maximum visibility for your research: over 100M website views per year

At BMC, research is always in progress.

Learn more biomedcentral.com/submissions 